SILICON

PHOTO-MULTIPLIERS

• Robert Klanner - University of Hamburg - robert.klanner@desy.de - DOI: https://doi.org/10.1051/epn/2019401

The measurement of single or few photons with a time resolution of $1 \mathrm{~ns}$ or below is an experimental technique with many applications in research, medicine and industry. Until recently, vacuum photo-multipliers (PMTs) dominated this field. In the 1990s avalanche photo-diodes came into use, and in the last decade solid-state photo-multipliers (SSPMs) have replaced PMTs in many applications. In most cases early samples, not yet optimised for the specific application, have been used. In the meantime industry and research institutes have significantly improved the performance of SSPMs.
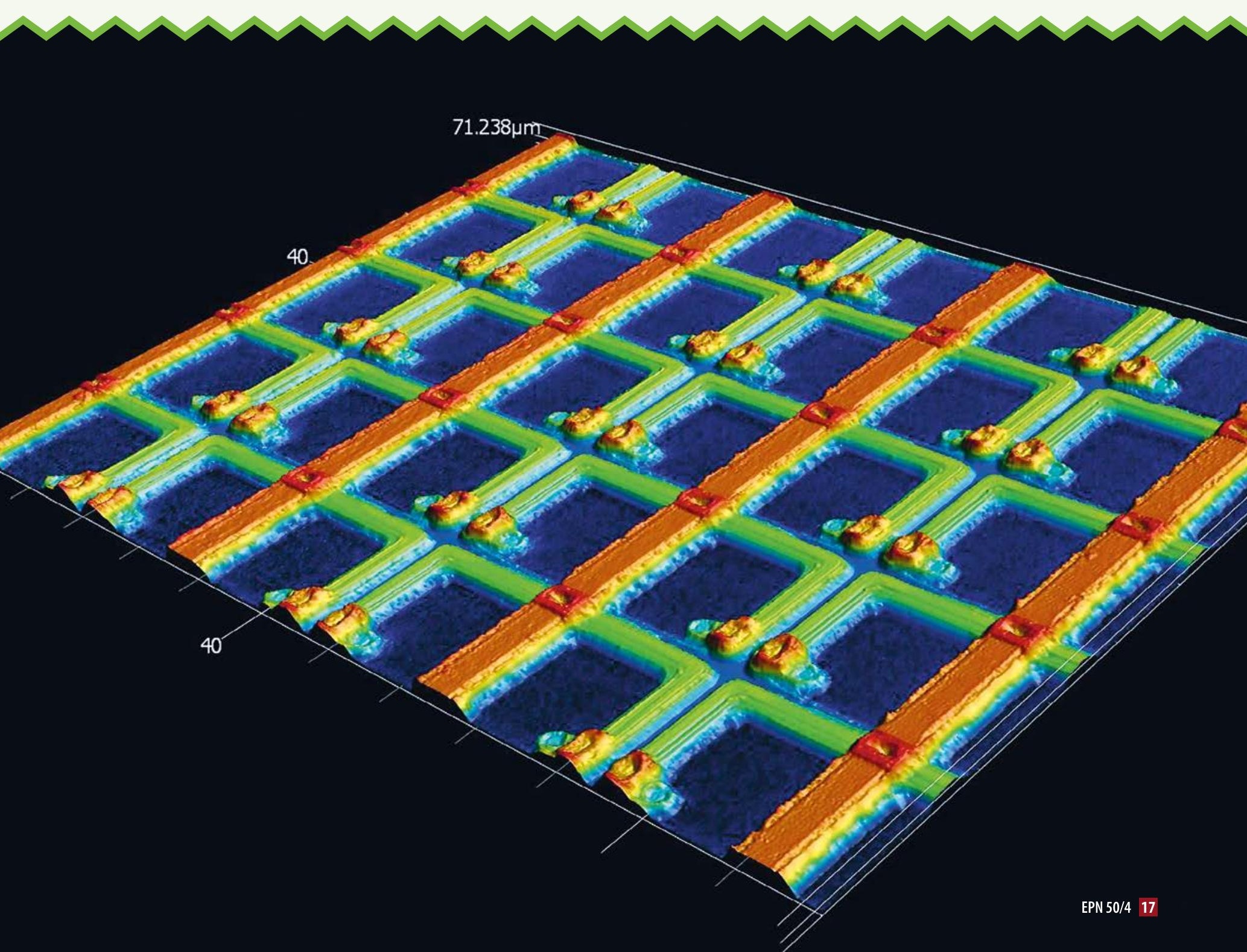


\section{Principles of Operation}

Silicon photo-multipliers (SiPMs) are arrays of hundreds to ten-thousands of Single-Photon Avalanche Diodes (SPADs) operated in Geiger mode above the break-down voltage. Every SPAD is connected to a quenching circuit - in most cases just a resistor - which stops the Geiger discharge. A photon, which converts in the SPAD into an electron-hole pair, can initiate a Geiger discharge resulting in a current pulse of charge $\mathrm{Q}$ (with $\mathrm{Q}$ = product of (SPAD capacitance) $\times \Delta \mathrm{V}$, where $\Delta \mathrm{V}$ is the difference of bias and quenching voltage). Typical gains (Q-values for a single Geiger discharge) are between $10^{5}$ and $10^{7}$. The SiPM output signal is the sum of all SPAD signals. As the signal of a single SPAD does not depend on the number of converting photons, SiPMs are digital detectors counting the number of SPADs with Geiger discharges. As shown in figure 1, the signals from a SiPM consist of narrow, well separated peaks corresponding to the different number of Geiger discharges.

One distinguishes analogue and digital SiPMs: In analogue SiPMs all SPADs are connected in parallel; in digital SiPMs every SPAD has its separate readout electronics. In the following we will concentrate on analogue SiPMs, as they are used in most applications today. However, recent progress in integrating SiPMs and 3D-CMOS technology shows the large potential of digital SiPMs for future innovative applications [2].

Figure 2 shows a specific example of the layout and the doping of a SPAD. A p ${ }^{+}$-epi-layer, which forms the high-field region, is grown on a $\mathrm{p}^{+}$-substrate. The diode is realised as an $\mathrm{n}^{+}$-implant on top of an additional $\mathrm{p}$-implant. The photons to be detected enter through the Anti-Reflecting-Coating (ARC). Between two SPADs additional $\mathrm{p}^{+}$-implants isolate the SPADs electrically. The $\mathrm{n}^{+}$-implants are connected via the quenching resistor to the top metal biasing lines, which connect the SPADs to the supply voltage. In order to optically separate two SPADs, trenches can be implemented as shown on the bottom.

An outstanding SiPM property is the high photondetection efficiency (PDE), which can be achieved. The optimisation depends on the wavelength of the light and

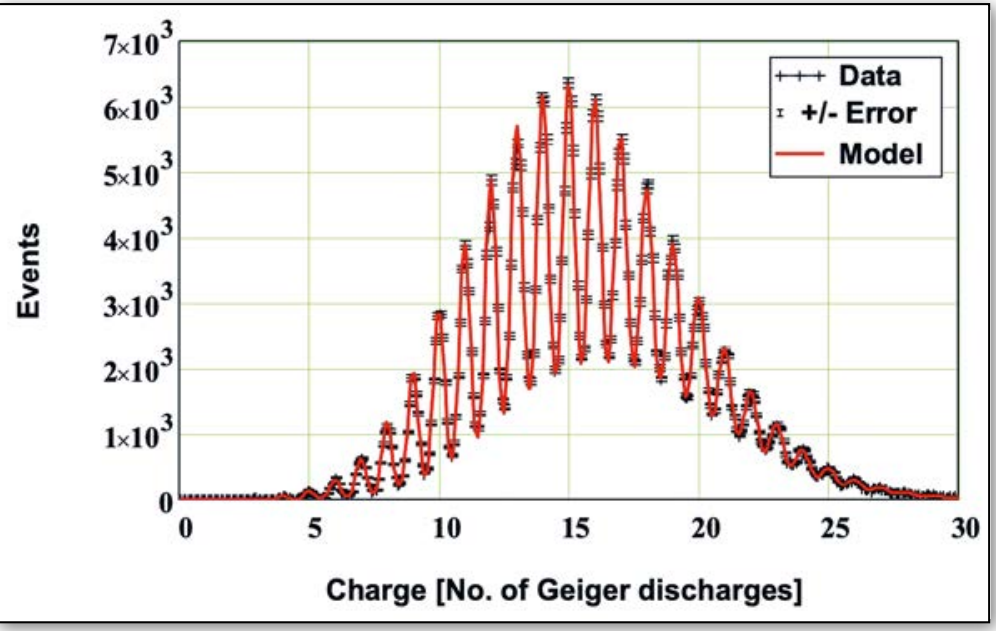

the SiPM operating temperature. The reason is that the light-absorption length in silicon changes from $10 \mathrm{~nm}$ for wavelengths between 75 and $350 \mathrm{~nm}$ to $10 \mu \mathrm{m}$ at $800 \mathrm{~nm}$, and that the ionisation rates for holes and electrons are quite different and strongly temperature dependent. Figure 3 shows as an example how the PDE can be optimised by changing the diode design. The PDE is proportional to the probability that an electron-hole pair causes a Geiger discharge and increases with bias voltage. The voltage above the Geiger breakdown voltage is given in the figure. An additional challenge, in particular for SPAD sizes as small as $10 \mu \mathrm{m} \times 10 \mu \mathrm{m}$, is achieving a high fill factor, the ratio of sensitive area to total SiPM area.

Different noise sources worsen the SiPM performance compared to ideal photo-detectors. As already a single charge can generate a Geiger discharge, the dark-count rate per unit area (DCR) of SiPMs is much larger than for PMTs. Typical DCRs at room temperature, where thermal generation dominates, are $100 \mathrm{kHz} / \mathrm{mm}^{2}$. Cooling significantly reduces the DCR until band-to-band tunnelling dominates. For specific designs DCRs below $10^{-2} \mathrm{~Hz} / \mathrm{mm}^{2}$ have been reached at cryogenic temperatures [1]. Irradiation by hadrons results in a dramatic increase of DCR: An increase by 7 orders of magnitude for neutron fluences exceeding $10^{13} \mathrm{~cm}^{-2}$ is observed.

Cross-talk between neighbouring SPADs and after-pulses are other effects, which degrade the SiPM performance. During the Geiger discharge hot charge carriers emit photons, which can convert in the silicon. If they convert in the SPAD of the Geiger discharge, they have no effect, as the SPAD has not yet charged up to the bias voltage. However, if they reach a neighbouring SPAD, they produce a quasi-prompt Geiger discharge. This can be prevented by an opaque trench, as shown in figure 2. Care also has to be taken that photons do not reach SPADs via external optical paths. Photons also convert in the non-depleted substrate, and charge carriers can reach a SPAD via diffusion and generate delayed noise pulses there. In addition, charge carriers trapped in defect states in the silicon band gap can trigger delayed Geiger discharges, when they are released.All these effects are proportional to the number of charges in the Geiger discharge, and therefore proportional to the SiPM gain. They can be described by an Excess-Charge-Factor and an Excess-Noise-Factor, which parametrise the increase of the mean signal and the worsening of the signal spread compared to a Poisson distribution for the primary Geiger discharges.

SiPMs essentially count the number of converting photons, and their response is non-linear once the number of Geiger discharges is not small compared to the number of SPADs. Even for SiPMs with as many as 10000 SPADs the dynamic range is significantly lower than for PMTs. Because of the recharging of the SPADs, the 


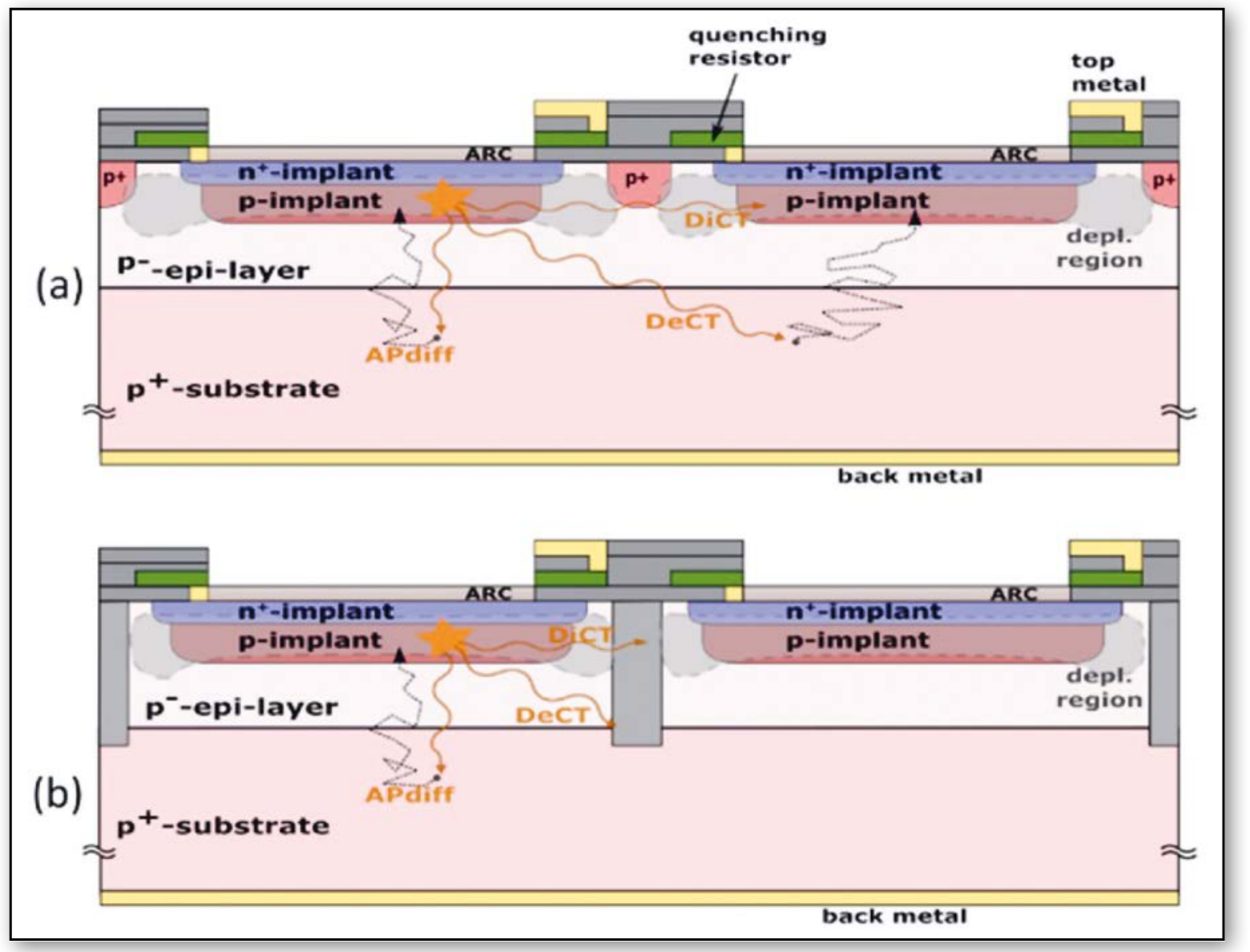

non-linearity depends on the arrival-time distribution of the photons, and its understanding is quite complex. In addition, the SPAD signal shapes change with the number of simultaneously generated electron-hole pairs, an effect which is not yet understood in any detail.

Given the high field and the $\mu \mathrm{m}$ depth of the amplification region, SiPMs are intrinsically fast detectors with excellent time resolution. Resolutions of 20 ps (FWHM) have been achieved and further improvements are expected.

As solid-state detectors SiPMs are very robust and do not show signs of degradation, even when exposed to high light intensities. Another advantage is that they are simple to use and their operating voltage is only a few tens of Volts. However, as the break-down voltage and thus the gain changes with temperature, SiPMs have to be operated either in a temperature stabilised environment or with temperature regulation. Several such systems have been developed. For many applications it is also important that SiPMs are not affected by magnetic fields.

\section{Examples of SiPM applications}

Already at the time of the first successful demonstrations of the SiPM concept in the late 1990s, the many possible applications had been realised [5]. However, it was also realised that different applications need different optimisations as discussed in [1]. Today SiPMs are used in fields as diverse as nuclear and particle physics, spectroscopy, medicine, biology, and LIDAR (Light Detection and Ranging). As it is not possible to cover all these applications in a short overview article, only three examples are given.

The combination of Magnetic Resonance Imaging (MRI), which provides exquisite anatomical information, with Positron Emission Tomography (PET) giving functional information, is a major step forward in medical instrumentation. Given the high magnetic fields required for MRI, solid state photo-detectors, which are immune to magnetic fields, are ideal to realise MRI/PET systems. In addition, the precise time measurement can be used to suppress backgrounds. By now a number of systems e.g. for whole-body and brain scanning using SiPMs have

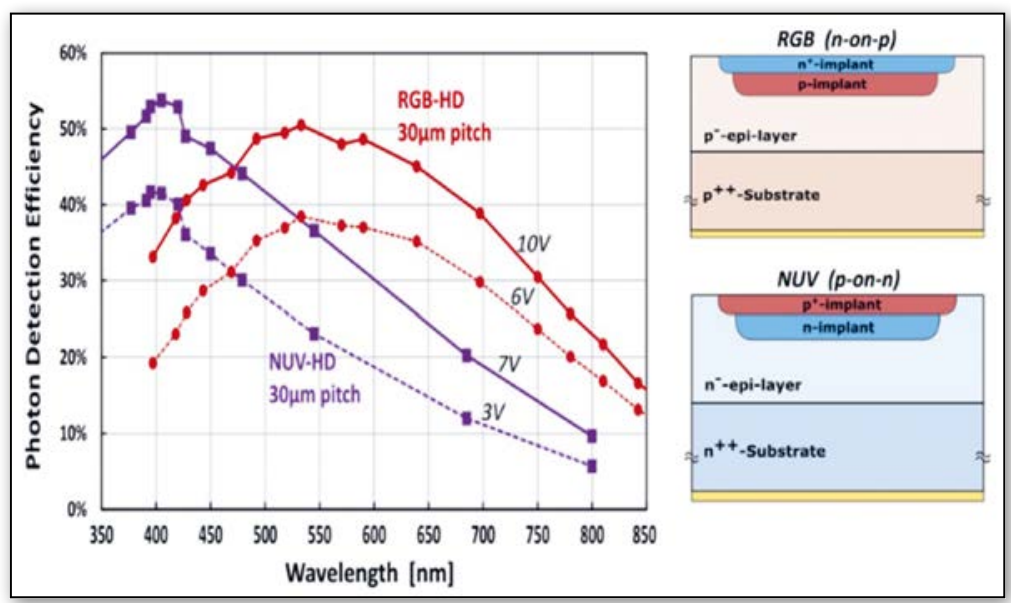

$4 \mathrm{FIG} .2:$

Cross-section of SPADs without (a) and with (b) trenches for optical isolation from [1]. Different sources of correlated noise, which are discussed in the text, are indicated. The depth of the $\mathrm{p}^{+}$-epi layer is one to a few $\mu \mathrm{m}$.

vFIG. 3:

Photon-detection efficiency optimised for different wavelengths and the corresponding SPAD designs from [1] 


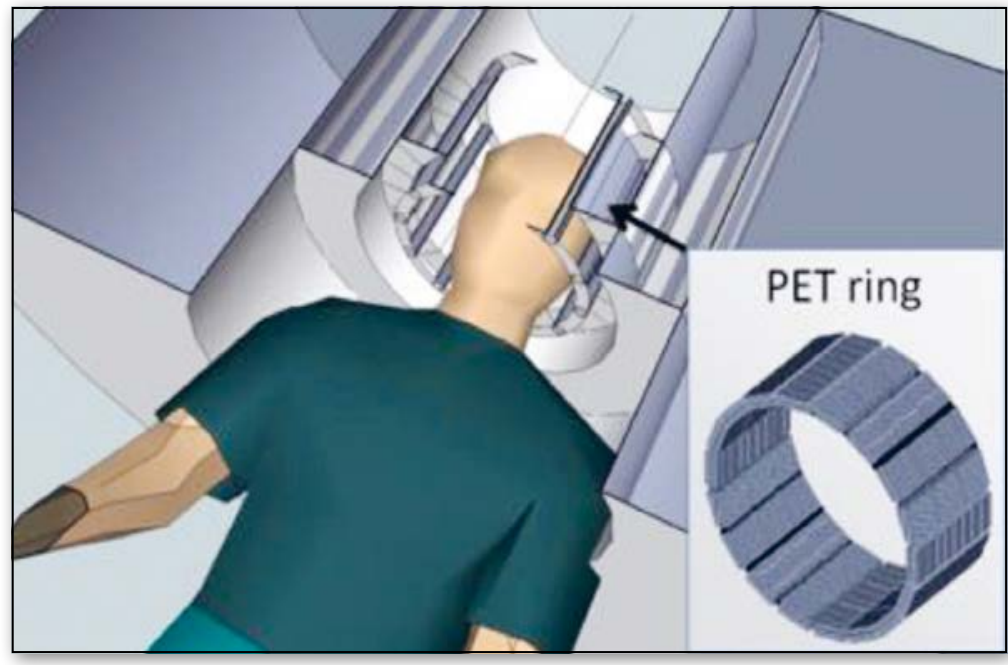

$\triangle F I G .4:$ been developed. In the innovative TRIMAGE project, Schematic Electro-Encephalo-Graphy is added to a PET/MRI scandrawing of the TRIMAGE scanner. The EEG cap is not shown [4]. ner as a tri-modality imaging tool for schizophrenia [4] Figure 4 show a schematic drawing of the TRIMAGE brain scanner.

The second example is Imaging Atmospheric Cherenkov Telescopes (IACT). They detect high energy gamma rays from the sky. With the discovery of more than 200 gamma-ray sources of galactic and extra-galactic origin, IACTs have contributed to better understand galactic nuclei, pulsars, black holes and the origin of cosmic rays So far IACT detectors have used PMTs. IACTs detect faint flashes of Cherenkov light from particle showers in the atmosphere and can only operate during nights. The backgrounds from air glow, diffuse starlight and even worse from the moon, represent major limitations, with respect to measurement time and photo-detection stability. The stability of SiPMs when exposed to background light make them candidates for this application. Their

\section{SUMMARY BOX}

Silicon-Photo-Multipliers are arrays of hundreds to ten thousands of Single-Photon-Avalanche-Photodiodes operated in quenched Geiger mode above the break-down voltage. Given their excellent performance, they now replace Vacuum-Photo-Multipliers in many applications. Their photon-detection efficiency can exceed $50 \%$ in a wide wavelength interval, they count single photons with a time resolution in the 20 ps (FWHM) range, operate at voltages of a few tens of volts, are insensitive to magnetic fields, are robust and do not deteriorate, even if exposed to strong light. However, their size is limited to about $10 \mathrm{~cm}^{2}$, their dynamic range is limited by the number of cells, and they have dark-count rates of tens to hundreds of $\mathrm{kHz} / \mathrm{mm}^{2}$ at room temperature. Their photon-counting resolution is influenced by optical cross-talk and afterpulsing. Developments in the last years resulted in major performance improvements and further progress is expected. use for IACTs has been pioneered by the construction of FACT [6]. The FACT camera consisting of 1440 SiPMs with 3660 SPADs each has a diameter of $40 \mathrm{~cm}$. It has been installed close to the MAGIC telescope at the Roque de los Muchachos observatory on the Island of La Palma in 2011, and tested by measuring the gamma-spectrum of the Crab-nebula between $250 \mathrm{GeV}$ and $16 \mathrm{TeV}$. The results are in perfect agreement with the measurement of the MAGIC telescope, demonstrating the reliable performance of this new technology. Based on this success, CTA, the large Cherenkov Telescope Array, plans to use SiPMs for their cameras.

Excellent timing resolution and high photon sensitivity make SiPMs prime candidates for LIDAR applications, including automatic driving. In [7] the performance of a SiPM is compared to a PMT for atmospheric LIDAR in the same setup. Already for a non-optimised SiPM an improvement of the signal-to-noise-ratio by about $50 \%$ for Aerosol observation up to a height of $3.35 \mathrm{~km}$ above ground level is observed. With optimised SiPMs significant improvements are expected.

\section{Conclusions}

Solid-State Photo-Multipliers are relatively new detectors for measuring single and multiple photons with excellent time resolution. Already the first generation has found numerous applications and has replaced classical vacuum-photomultipliers in many measurement tasks. In the meantime, intensive $\mathrm{R} \& \mathrm{D}$ in academia and industry has resulted in significant performance improvements, and the use of solid-state photo-multipliers is expanding rapidly.

\section{About the Author}

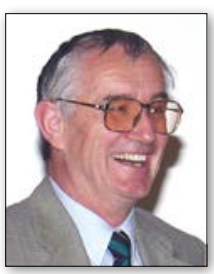

Robert Klanner, Prof. emeritus at Hamburg University. Experimental particle physicist, who contributed to the development of silicon sensors and calorimeters and their use in experiments.

\section{References}

[1] F. Acerbi et al. , Instruments 3, 15 (2019).

[2] E. Charbon, C. Bruschini, and Lee Myung-Jae, Circuits and Systems (ICECS), 1 (2018).

[3] V. Chmill et al., Nucl. Instrum. Methods Phys. Res. A 854, 70 (2017).

[4] A. Del Guerra et al., Eur. Psychiatry 50, 7 (2018).

[5] B. Dolgoshein et al., Nucl. Instrum. Methods Phys. Res. A 563, 368 (2006).

[6] Noethe M. et al., Performance of the First Cherenkov Telescope Observing with SiPMs. POS (ICRC 2017), 791 (2017).

[7] J. Riu et al., Optics Letters 37, 7, 1229(2012). 